\title{
Utilização da Tabela de cronologia de mineralização dental de Nicodemo, Moraes e Médici Filho na estimativa da idade de paraibanos*
}

\section{The use of the Nicodemo, Moraes e Médici Filho Table of dental mineralization chronology to estimate the Paraibanos' age}

\author{
Marina Barrêto Pereira Moreno'; Tércio José Pereira Pontes'; \\ Patrícia Moreira Rabello
}

\begin{abstract}
Moreno MBP; Pontes TJP; Rabello PM. Utilização da Tabela de cronologia de mineralização dental de Nicodemo, Moraes e Médici Filho na estimativa da idade de paraibanos. Saúde, Ética \& Justiça. 2014;19(1);35-44.

RESUMO: Objetivo: Verificar o índice de acerto da idade cronológica de jovens quando utilizada a tabela de Nicodemo, Moraes e Médici Filho na estimativa da idade por meio de radiografia panorâmica de paraibanos. Metodologia: A amostra foi composta por 94 imagens de radiografias panorâmicas de indivíduos entre 10 e 25 anos. Utilizou-se a tabela de mineralização descrita no estudo de NOLLA de acordo com a cronologia de mineralização dos dentes permanentes no Brasil criada por Nicodemo, Moraes e Médici Filho. O tipo de estudo foi o transversal e cego. Para análise dos dados, foram obtidas distribuições absolutas e percentuais e foi utilizado o teste Qui-quadrado de Pearson. O nível de significância utilizado nas decisões dos testes estatísticos foi de 5\%. Resultados: O percentual de acerto nesta faixa etária foi maior quando se analisaram apenas 4 dentes por radiografia panorâmica $(81,9 \%)$ em relação à análise de 16 (4,3\%). A análise baseada na imagem dos 3 os molares apresentou o maior percentual de acerto $(66 \%)$ em relação à avaliação dos demais dentes. Não houve diferença estatística entre o percentual de acerto quando comparados os resultados para homens e mulheres do grupo total $(\mathrm{p}=0,479)$. O percentual de acerto foi maior quando utilizou-se a imagem da amostra entre 10 e 15 anos (94,4\%) em relação aos com mais de 15 anos (65\%), apresentando diferença estatisticamente significante (p>0,001). Conclusão: Os métodos de estimativa da idade utilizando a tabela de Nicodemo, Moraes e Médici Filho propostos por este estudo demonstraram percentuais de acerto baixos quando da avaliação de 16 dentes. Os resultados foram mais satisfatórios com a utilização de apenas 4 dentes na avaliação, principalmente na faixa etária dos 10 aos 15 anos. Em termos da prática odontolegal, os resultados apresentavam intervalos etários muito amplos, o que inviabilizaria o uso deste método isoladamente na amostra estudada. Na busca de um método de estimativa de idade para responder à solicitação da Justiça, os Odontolegistas devem associar mais de uma técnica e, com isto, oferecer conclusões mais precisas.
\end{abstract}

DESCRITORES: Odontologia Legal; Determinação da Idade pelos Dentes; Radiologia.

\footnotetext{
* O texto é inédito. Aprovado por pelo Comitê de Ética e Pesquisa da Universidade Federal da Paraíba CEP-CCS, no dia 09/07/2008, protocolo $\mathrm{n}^{\circ} 0283$.

1. Graduados em Odontologia pela Universidade Federal da Paraíba.

2. Professora Doutora Adjunta do Departamento de Clínica e Odontologia Social da UFPB.

Endereço para correspondência: Universidade Federal da Paraíba, Centro de Ciências da Saúde - Campus I, Departamento de Odontologia Clínica e Social. Cidade Universitária - Campus I. CEP: 58059-900 - Joao Pessoa, PB - Brasil. E-mail: marinabpmoreno@hotmail.com
} 


\section{INTRODUÇ̃̃̃O}

A perícia é um procedimento especial de constatação, prova ou demonstração científica ou técnica, relacionado com a veracidade de uma situação ou análise ${ }^{1}$. As perícias odontolegais podem ser conceituadas como operações destinadas a ministrar esclarecimentos técnicos à Justiça, por meio da elaboração de documentos odontolegais autos, laudos e pareceres - os quais funcionarão como provas técnicas a serem analisadas pelo juiz no processo ${ }^{2}$.

Dentre os processos de identificação do indivíduo, a estimativa da idade é um dos mais utilizados pelos peritos. Ela pode ser feita tanto em vivos quanto em mortos (corpos ou ossadas). O diagnóstico de idade de pessoas vivas geralmente avalia a probabilidade de a pessoa ter atingido uma idade juridicamente relevante. No caso de cadáveres e esqueletos, é utilizada para auxiliar na identificação do indivíduo, quando o reconhecimento não é possível por outros métodos, usualmente em situações de mutilação, ausência de ossos, estágio avançado de decomposição ou mesmo, carbonização ${ }^{3}$.

São várias as metodologias para a estimativa de idade e a escolha dependerá das circunstâncias e de quão preciso o diagnóstico da idade deve ser no caso em apreço ${ }^{4}$. Existem técnicas que utilizam dados gerais como estatura, peso e presença de rugas, e outras avaliam caracteres sexuais secundários (presença e coloração de pelos pubianos e axilares), desenvolvimento de genitália externa; presença de mamas; desenvolvimento ósseo e desenvolvimento dentário ${ }^{5}$.

Os dentes, mesmo estando sujeitos a fatores intrínsecose extrínsecos que alteram seu desenvolvimento, são relativamente estáveis quando comparados a outras estruturas do corpo humano, já que reagem de maneira peculiar aos estímulos externos 5 .

Desde a vida intrauterina até a erupção dos 3 os molares, uma sucessão de estágios, que se encontram perfeitamente identificados e caracterizados na literatura, ocorre na dentadura dos seres humanos. Pela pouca variação do desenvolvimento dentário entre as diversas etnias, sua avaliação permite o cálculo da idade do indivíduo com satisfatória precisão. Nesse caso, é valiosa sua contribuição na perícia para estimativa de idade em crianças e adolescentes, pois quanto mais jovem o indivíduo, maior o número de informações que se pode obter da análise da dentição, em razão do maior número de dentes em formação ${ }^{1}$.

De fato, a avaliação da cronologia da mineralização dos dentes é um dos métodos mais comuns para se estabelecer a idade em seres humanos, sejam estes vivos, mortos ou em fase de esqueletização $0^{6}$. Para melhor compreensão, pode-se classificar a evolução dentária em períodos de desenvolvimentos e etapas: lâmina dentária, calcificação ou mineralização dos dentes, cronologia da erupção e modificações tardias ${ }^{3}$.

O estudo das etapas da evolução dentária pode ser realizado tanto pelo método direto, que consiste na análise clínica, em que se verificam o número de elementos dentários, a sequência eruptiva e o estado geral dos dentes; quanto pelo método indireto realizado por meio do estudo de exames radiográficos. Schimidt ${ }^{3}$ afirma que estes métodos fornecem elementos suficientes para a aferição do estágio de desenvolvimento do indivíduo, desde o embrião de 6 semanas até a idade de 18 e 21 anos.

O método radiográfico é considerado mais fiel do que o clínico porque se baseia na mineralização dos elementos dentários, independentemente da sua erupção, esta, sujeita a fatores ambientais e locais. Baseia-se na análise de imagens radiográficas em comparação com valores estabelecidos em tabelas com dados padronizados ${ }^{7}$.

Estudos em diferentes populações foram realizados a fim de estimar a idade de indivíduos com base no desenvolvimento dentário. A tabela de Nicodemo, Moraes e Médici Filho, foi desenvolvida no estado de São Paulo com este fim. Os autores perceberam que as tabelas estrangeiras descritas na literatura não eram compatíveis com a amostra brasileira e perceberam a necessidade da avaliação da idade dentária a partir de uma tabela com padrões nacionais ${ }^{8}$.

Dessa forma, o presente estudo teve por objetivo correlacionar a idade cronológica de indivíduos paraibanos de 10 a 25 anos com as idades sugeridas na tabela de Nicodemo, Moraes e Médici Filho, tendo por base os estágios de mineralização dental.

\section{METODOLOGIA}

Trata-se de estudo do tipo transversal e cego. A amostra total foi composta por 94 imagens de radiografias panorâmicas, sendo 46 radiografias de indivíduos do sexo masculino e 48 radiografias de indivíduos de sexo feminino. As radiografias foram avaliadas por um único pesquisador. Todas as radiografias foram realizadas no mesmo equipamento, no ano de 2010, digitalizadas e cedidas por uma clínica de radiologia da cidade de João Pessoa, Paraíba. Foram incluídas as radiografias de indivíduos com idade entre 10 e 25 anos, nascidos em diferentes cidades do estado da Paraíba e descartadas as radiografias que possuíam ausência de vários elementos dentários, quando o mesmo elemento dentário estivesse ausente em ambos os lados de um mesmo arco, principalmente segundos e terceiros molares, bem como aquelas com qualidade ruim, ou seja, falta de nitidez, contraste e pouca visibilidade. Este estudo foi aprovado pelo Comitê de Ética do Centro de Ciências da Saúde da Universidade Federal da Paraíba.

A calibração consistiu na análise de 6 radiografias 
panorâmicas, previamente selecionadas, e sua posterior comparação com a tabela dos estágios de mineralização dos elementos dentários de Nolla, modificada por Nicodemo, Moraes e Médici Filho. Após 8 dias, as radiografias foram reavaliadas para se obter a concordância intraexaminador. O valor de kappa foi 0,94 , com intervalo de confiança 0,86 a 1,00 .

Os dados foram coletados através de um estudo cego, no qual se estimou a idade de homens e mulheres, exclusivamente por meio da avaliação imaginológica. Cada amostra foi analisada em 2 avaliações distintas, em duas fases diferentes.

Na primeira avaliação, em cada radiografia, foram analisados os estágios de mineralização dental de 16 dentes, com o critério de melhor visualização, sendo 8 superiores e 8 inferiores. O estágio de mineralização em que cada dente se encontrava foi determinado por meio da tabela de Nicodemo, Moraes e Médici Filho, utilizando a descrição na faixa de meses, com limites mínimo e máximo, para todos os dentes analisados. Após essa etapa, somaram-se todas as idades mínimas e dividiu-se pelo número de dentes analisados. $\mathrm{O}$ mesmo procedimento foi realizado usando-se a idade máxima. Essa metodologia foi repetida em todas as 94 radiografias panorâmicas.

$\mathrm{Na}$ segunda, foram avaliados apenas 4 dentes em cada radiografia, 1 segundo e 1 terceiro molar por arcada, sem levar em consideração o hemiarco em que cada dente se encontrava. Determinados os estágios de mineralização de cada dente pela tabela cronológica de Nicodemo, Moraes e Médici Filho, definiu-se a faixa etária do indivíduo observando a menor idade mínima e a maior idade máxima de todos os dentes examinados.

Após comparação dos resultados com a idade cronológica, realizou-se a análise estatística descritiva dos estágios de calcificação (média, desvio padrão, gráficos e porcentuais dos estágios), utilizando-se o SPSS (Statistical Package for the Social Sciences) for Windows (versão 17), e avaliou-se a variabilidade dos segundos e terceiros molares, verificando o grau de aplicabilidade e veracidade das informações etárias obtidas na pesquisa em relação às idades reais.

Para a análise dos dados, foram obtidas distribuições absolutas e percentuais e foi utilizado o teste Qui-quadrado de Pearson. O nível de significância utilizado nas decisões dos testes estatísticos foi de $5 \%$.

\section{RESULTADOS}

No primeiro estudo, dos 94 pacientes, em apenas $4(4,3 \%)$ houve acerto na faixa etária quando analisados os 16 dentes. Ao contrário, na segunda fase do trabalho, quando apenas 4 dentes foram pesquisados, obteve-se 77 $(81,9 \%)$ de acerto do total da amostra, independentemente do dente avaliado, quando se comparou a idade estimada com a idade cronológica do indivíduo.

Na Tabela 1 estão os resultados do acerto por grupo de dente ou por dente no arco superior. A Tabela 2 apresenta os resultados no arco inferior e na, Tabela 3 , os resultados independentemente do arco. Na Tabela 1 é possível verificar que o maior percentual de acerto foi registrado para o 3 os molares superiores $(63,8 \%)$; não houve acerto na avaliação dos incisivos centrais superiores (ICS), incisivos laterais superiores (ILS) e $1^{\text {os }}$ molares superiores (MS). Nos demais dentes, os percentuais variaram de $16,0 \%$ ( 2 os pré-molares superiores, PMS) a $26,6 \%$ ( $2^{\text {os }}$ molares superiores, MS).

TABELA 1 - Distribuição numérica e percentual dos acertos e erros entre a idade estimada e a real observados por mineralização de 8 elementos dentários superiores, de indivíduos entre 10 e 25 anos, em João Pessoa-PB, 2012 (n=94)

\begin{tabular}{|c|c|c|c|c|c|c|}
\hline \multirow[t]{2}{*}{ Dente } & \multicolumn{2}{|c|}{ Acerto } & \multicolumn{2}{|c|}{ Erro } & \multicolumn{2}{|c|}{ Não avaliado* } \\
\hline & $\mathrm{N}$ & $\%$ & $\mathrm{~N}$ & $\%$ & $\mathrm{n}$ & $\%$ \\
\hline - ICS & - & - & 93 & 98,9 & 1 & 1,1 \\
\hline - ILS & - & - & 92 & 97,9 & 2 & 2,1 \\
\hline - $\mathrm{CS}$ & 23 & 24,5 & 71 & 75,5 & - & - \\
\hline - $1^{\circ} \mathrm{PMS}$ & 22 & 23,4 & 69 & 73,3 & 3 & 3,2 \\
\hline - $2^{\circ} \mathrm{PMS}$ & 15 & 16,0 & 78 & 83,0 & 1 & 1,1 \\
\hline - $1^{\circ} \mathrm{MS}$ & - & - & 94 & 100,0 & - & - \\
\hline - $2^{\circ} \mathrm{MS}$ & 25 & 26,6 & 69 & 73,4 & - & - \\
\hline - $3^{\circ} \mathrm{MS}$ & 60 & 63,8 & 34 & 36,2 & - & - \\
\hline
\end{tabular}

*Elemento dentário que não foi possível observar com clareza nas radiografias

Resultados similares podem ser observados na Tabela 2, para o arco inferior. O maior percentual de acerto foi registrado para os 3 os molares inferiores $(68,1 \%)$, sendo que não houve acerto na análise dos incisivos centrais inferiores (ICI), incisivos laterais inferiores (ILI) e $1^{\underline{\text { os }}}$ molares inferiores (MI). Nos demais dentes, a variação de acerto situou-se entre $25,5 \%$ e $31,9 \%$. 
Moreno MBP et al. Utilização da Tabela de cronologia de mineralização dental de Nicodemo, Moraes e Médici Filho.

TABELA 2 - Distribuição numérica e percentual dos acertos e erros entre a idade estimada e a real observados por mineralização de 8 elementos dentários inferiores de indivíduos entre 10 e 25 anos, em João Pessoa-PB, 2012 (n=94)

\begin{tabular}{|c|c|c|c|c|c|c|}
\hline \multirow[t]{2}{*}{ Dente } & \multicolumn{2}{|c|}{ Acerto } & \multicolumn{2}{|c|}{ Erro } & \multicolumn{2}{|c|}{ Não avaliado* } \\
\hline & $\mathrm{N}$ & $\%$ & $\mathrm{~N}$ & $\%$ & $\mathrm{~N}$ & $\%$ \\
\hline - ICS & - & - & 92 & 97,9 & 2 & 2,1 \\
\hline - ILS & - & - & 94 & 100,0 & - & - \\
\hline - $\mathrm{CS}$ & 25 & 26,6 & 69 & 73,4 & - & - \\
\hline - $1^{\circ} \mathrm{PMS}$ & 24 & 25,5 & 69 & 734 & 1 & 1,1 \\
\hline - $2^{\circ} \mathrm{PMS}$ & 24 & 25,5 & 70 & 74,5 & - & - \\
\hline$\cdot 1^{\mathrm{o}} \mathrm{MS}$ & - & - & 94 & 100,0 & - & - \\
\hline$\cdot 2^{\circ} \mathrm{MS}$ & 30 & 31,9 & 64 & 68,1 & - & - \\
\hline - $3^{\circ} \mathrm{MS}$ & 64 & 68,1 & 30 & 31,9 & - & - \\
\hline
\end{tabular}

*Avaliação do elemento prejudicada pela nitidez nas radiografias

As Tabelas 3 a 7 apresentam os resultados do acerto por dente no grupo total e estratificados por sexo e por faixa etária da segunda fase do estudo, ou seja, da análise de apenas 4 elementos por radiografia. Na Tabela
3 é possível verificar que os maiores percentuais de acertos foram observados para os $3^{\text {os }}$ molares $(61,7 \%)$ e variaram de $27,7 \%$ a $30,9 \%$ para os $2^{\circ} \mathrm{S} \mathrm{MS}$ e $2^{\circ} \mathrm{s} \mathrm{MI}$, respectivamente.

TABELA 3 - Distribuição numérica e percentual dos acertos e erros entre a idade estimada e a real quando da análise da mineralização de 4 elementos dentários de um mesmo indivíduo: segundos e terceiros molares, inferiores e superiores, João PessoaPB, $2012(n=94)$

\begin{tabular}{lllll}
\hline Dente & Acerto & & Erro & \\
\hline & $\mathrm{N}$ & $\%$ & $\mathrm{~N}$ & $\%$ \\
\hline$\cdot 2^{\mathrm{o}} \mathrm{MS}$ & 26 & 27,7 & 68 & 72,3 \\
$\cdot 3^{\text {o }} \mathrm{MS}$ & 58 & 61,7 & 36 & 38,3 \\
$\cdot 2^{\mathrm{o}} \mathrm{MI}$ & 29 & 30,9 & 65 & 69,1 \\
$\cdot 3^{\text {o } \mathrm{MI}}$ & 58 & 61,7 & 36 & 38,3 \\
\hline
\end{tabular}

Os resultados da Tabela 4 indicam o percentual de acerto da faixa etária para o total (4 dentes) das 46 radiografias de indivíduos do sexo masculino e 48 radiografias de indivíduos de sexo feminino. Não houve diferença estatisticamente significativa entre os grupos, muito embora numericamente tenha havido um percentual mais elevado de acerto quando da análise de radiografias das mulheres $(84,8 \%)(\mathrm{p}>0,05)$.

TABELA 4 - Distribuição numérica e percentual, por sexo, dos acertos e erros entre a idade estimada e a real quando da análise da mineralização de 4 elementos dentários do mesmo indivíduo, João Pessoa-PB, 2012 ( $\mathrm{n}=48$ para o sexo masculino e $\mathrm{n}=46$ para o sexo feminino)

\begin{tabular}{llllll}
\hline \multicolumn{7}{c}{ Gênero } \\
\hline Grupo total & Masculino & \multicolumn{3}{c}{ Feminino } & Valor de $\mathrm{p}$ \\
\hline Acerto & $\mathrm{N}$ & $\%$ & $\mathrm{~N}$ & $\%$ & $\mathrm{p}^{(1)}=0,479$ \\
Erro & 38 & 79,2 & 39 & 84,8 & \\
\hline
\end{tabular}

(*): Diferença significativa ao nível de 5,0\%.

(1): Através do teste Qui-quadrado de Pearson. 
Na Tabela 5 é possível verificar que a maior diferença percentual de acertos ocorreu nos 3 os MIs, que tiveram valores mais elevados na análise das radiografias de indivíduos do sexo feminino (73,9\%). A estimativa da idade proporcionada por estes dentes demonstrou diferença significativa entre gêneros $(\mathrm{p}<0,05)$. Os dados observados na tabela 6 mostram que o percentual de acertos foi bem mais elevado entre os pesquisados que tinham 10 a 15 anos $(94,4 \%)$ do que entre os com mais de 15 anos $(65,0 \%)$ e se comprova diferença significativa entre as faixas etárias em relação ao percentual de acertos $(\mathrm{p}<0,001)$.

TABELA 5 - Distribuição numérica e percentual por sexo dos acertos e erros entra a idade estimada e a real observados por mineralização dos segundos e terceiros molares, inferior e superior, João Pessoa-PB, 2012

\begin{tabular}{|c|c|c|c|c|c|}
\hline \multicolumn{6}{|c|}{ Sexo } \\
\hline \multirow[t]{2}{*}{ Dente } & \multicolumn{2}{|c|}{ Masculino } & \multicolumn{2}{|c|}{ Feminino } & \multirow[t]{2}{*}{ Valor de $\mathrm{p}$} \\
\hline & $\mathrm{N}$ & $\%$ & $\mathrm{n}$ & $\%$ & \\
\hline \multicolumn{6}{|l|}{$\cdot 2^{\circ} \mathrm{MS}$} \\
\hline Acerto & 12 & 25,0 & 14 & 30,4 & $\mathrm{p}^{(1)}=0,556$ \\
\hline Erro & 36 & 75,0 & 32 & 69,6 & \\
\hline \multicolumn{6}{|l|}{$\cdot 3^{\circ} \mathrm{MS}$} \\
\hline Acerto & 31 & 64,6 & 27 & 58,7 & $\mathrm{p}^{(1)}=0,557$ \\
\hline Erro & 17 & 35,4 & 19 & 41,3 & \\
\hline \multicolumn{6}{|l|}{ - $2^{\circ} \mathrm{MI}$} \\
\hline Acerto & 14 & 29,2 & 15 & 32,6 & $\mathrm{p}^{(1)}=0,718$ \\
\hline Erro & 34 & 70,8 & 31 & 67,4 & \\
\hline \multicolumn{6}{|l|}{$\cdot 3^{\circ} \mathrm{MI}$} \\
\hline Acerto & 24 & 50,0 & 34 & 73,9 & $\mathrm{p}^{(1)}=0,017^{*}$ \\
\hline Erro & 24 & 50,0 & 12 & 26,1 & \\
\hline
\end{tabular}

(*): Diferença significativa ao nível de 5,0\%.

(1): Através do teste Qui-quadrado de Pearson.

TABELA 6 - Distribuição numérica e percentual por faixa etária dos acertos e erros entre a idade estimada e a real quando da análise da mineralização de 4 elementos dentários do mesmo indivíduo, João Pessoa-PB, 2012

\begin{tabular}{lccccc}
\hline \multicolumn{5}{c}{ Faixa etária (anos) } \\
\hline Grupo total & \multicolumn{2}{c}{10 a 15} & \multicolumn{2}{c}{ Mais de 15 ou mais } & Valor de $\mathrm{p}$ \\
\hline Acerto & $\mathrm{N}$ & $\%$ & $\mathrm{~N}$ & $\%$ & \\
\hline Erro & 51 & 94,4 & 26 & 65,0 & $\mathrm{p}^{(1)}>0,001^{*}$ \\
\hline - TOTAL & 3 & 5,6 & 14 & 35,0 & \\
\hline
\end{tabular}

(*): Diferença significativa ao nível de 5,0\%.

(1): Através do teste Qui-quadrado de Pearson.

A Tabela 7 mostra que o percentual de acertos foi bem mais elevado entre os indivíduos que tinham de 10 a 15 anos do que entre os que tinham mais de 15 anos, uma vez que não houve acerto quando avaliados os segundos molares neste grupo. Foram registradas diferenças significativas entre as duas faixas etárias para cada um dos dentes $(\mathrm{p}<0,05)$. 
Moreno MBP et al. Utilização da Tabela de cronologia de mineralização dental de Nicodemo, Moraes e Médici Filho.

TABELA 7 - Distribuição numérica e percentual por faixa etária dos acertos e erros entre a idade estimada e a real observados por mineralização dos segundos e terceiros molares, inferior e superior, João Pessoa-PB, 2012

\begin{tabular}{|c|c|c|c|c|c|}
\hline \multicolumn{6}{|c|}{ Faixa etária (anos) } \\
\hline Dentes & \multicolumn{2}{|c|}{10 a 15} & \multicolumn{2}{|c|}{ Mais de 15 anos } & \multirow{2}{*}{ Valor de $\mathrm{p}$} \\
\hline & $\mathrm{N}$ & $\%$ & $\mathrm{~N}$ & $\%$ & \\
\hline \multicolumn{6}{|l|}{ - $2^{\circ} \mathrm{MS}$} \\
\hline Acerto & 26 & 48,1 & - & - & $\mathrm{p}^{(1)}>0,001^{*}$ \\
\hline Erro & 28 & 51,9 & 40 & 100,0 & \\
\hline \multicolumn{6}{|l|}{$\cdot 3^{\circ} \mathrm{MS}$} \\
\hline Acerto & 38 & 70,4 & 20 & 50,0 & $\mathrm{p}^{(1)}=0,045^{*}$ \\
\hline Erro & 16 & 29,6 & 20 & 50,0 & \\
\hline \multicolumn{6}{|l|}{ - $2^{\circ} \mathrm{MI}$} \\
\hline Acerto & 29 & 53,7 & - & - & $\mathrm{p}^{(1)}>0,001 *$ \\
\hline Erro & 25 & 46,3 & 40 & 100,0 & \\
\hline \multicolumn{6}{|l|}{$\cdot 3^{\circ} \mathrm{MI}$} \\
\hline Acerto & 38 & 70,4 & 20 & 50,0 & $\mathrm{p}^{(1)}=0,045^{*}$ \\
\hline Erro & 16 & 29,6 & 20 & 50,0 & \\
\hline
\end{tabular}

(*): Diferença significativa ao nível de 5,0\%.

(1): Através do teste Qui-quadrado de Pearson.

\section{DISCUSSÃO}

Diversos métodos radiográficos para estimativa de idade na odontologia foram desenvolvidos, considerando características específicas das populações sendo estudadas. Assim, a aplicação indistinta de um método desenvolvido para uma população em outra, com características genéticas e comportamentais distintas, pode não ser adequada. Muitos autores, dentre eles Saliba ${ }^{9}$, acreditam que o método de Nolla ${ }^{10}$ seja eficiente e aplicável neste sentido, porém, por não apresentar uma representação gráfica de fácil interpretação para a determinação da idade real, tem sua aplicação dificultada, assim como o método de Dermirjian, Goldstein e Tanner ${ }^{11-14}$. Outro método conhecido, porém muito criticado, é o de Gustafson e $\mathrm{Koch}^{15}$, pois sua utilização para a determinação de idade para fins de imputabilidade é prejudicada, uma vez que exclui os terceiros molares na sua avaliação.

Para solucionar essa problemática, Nicodemo, Moraes e Médici Filho ${ }^{16}$, baseados nos estágios de mineralização de Nolla, transformaram os 10 estágios em apenas 8 e desenvolveram uma tabela em meses que associa graficamente os valores referidos por Nolla à idade máxima e mínima em meses para a caracterização do estágio para cada grupo de elementos dentários. Esta tabela foi aplicada em população leucoderma no estado de São Paulo.
De acordo com o IBGE ${ }^{17}$, verificam-se no estado de São Paulo 3 grupos de cor ou raça predominantes: $64,4 \%$ da população se autodeclarou branca, 5,8\% preta e $28,3 \%$ parda. Já na Paraíba observa-se uma distribuição de 36,4\% que se autodeclarava branca, 4,9\% preta e $58,4 \%$ parda. É importante destacar que se trata de uma declaração fornecida pelo próprio entrevistado e que esta percepção pode variar entre os indivíduos. Apesar de São Paulo e Paraíba se situarem em regiões diferentes do país com hábitos culturais, condições de saúde e hábitos alimentares distintos, essas diferenças ainda são menores quando comparadas a outros países na questão da etnia e hábitos culturais.

Fatores que afetam a formação, desenvolvimento e erupção dos dentes são difíceis de serem detectados ${ }^{18}$, mas acredita-se que variáveis ambientais, nutricionais $\mathrm{e}$ étnicas podem interferir no desenvolvimento dentário ${ }^{19}$. Existem dúvidas sobre como a origem étnica influencia a mineralização, entretanto sabe-se que essa diferença existe $^{20}$ e apesar do Brasil ser um país com extenso território, apresentar uma população miscigenada e diferentes hábitos culturais e alimentares entre as regiões, que poderiam influenciar no desenvolvimento da população, essas diferenças parecem ser menores do que quando comparadas com populações de outros países. Por isso optou-se, no presente estudo, pela utilização de uma tabela desenvolvida para uma população brasileira. 
A idade cronológica de um indivíduo não necessariamente coincide com sua idade de maturação. O desenvolvimento esquelético pode estar atrasado ou avançado em vários graus de divergência com sua idade cronológica real. Já a idade biológica envolve o desenvolvimento do corpo em relação à maturidade, engloba alterações anatômicas, dentais e maturação esquelética, desenvolvimento de características sexuais secundárias, função hormonal e atividade enzimática, uma vez que esses mecanismos podem ser influenciados pela genética, por fatores socioeconômicos, ambientais, nutricionais e por condições relacionas ao gênero do indivíduo. No presente estudo não foram levantados dados quanto a alterações de gestação, nutrição e nível socioeconômico da amostra estudada, o que pode explicar a variação dos resultados obtidos ${ }^{21}$.

O presente estudo se fundamentou no trabalho de Nicodemo, Moraes e Médici Filho ${ }^{16}$ para observar a aplicabilidade deste método na cidade de João Pessoa, PB. Foram analisadas radiografias panorâmicas de jovens na faixa etária de 10 a 25 anos e constatou-se que os incisivos e os primeiros molares completaram sua mineralização muito precocemente: no máximo com 116 meses todos esses dentes já estavam com seus ápices fechados. Por esse motivo a aplicação do método de Nolla ${ }^{10}$ foi ineficaz nessa faixa etária, obtendo apenas $4,3 \%$ de acerto da idade real quando estudados os 16 elementos dentais.

Para estimar a idade de uma pessoa através dos elementos dentais, quanto mais jovem o indivíduo, haverá um maior número de dentes em estágios distintos, de modo que a estimativa será mais fácil e seu resultado mais próximo da idade cronológica. A pesquisa mostrou que o percentual de acertos foi bem mais elevado entre os pesquisados que tinham de 10 a 15 anos do que entre os com mais de 15 anos, o que demonstra a maior dificuldade em estimar a idade de indivíduos entre 15 a 18 anos, que é a faixa etária que mais necessita de estimativa dentro dos institutos médico-legais devido à necessidade de se distinguir entre menores infratores e adultos que, em flagrante delito, não portando documentos, simulem idade inferior para não sofrer punições. No entanto, a mineralização do terceiro molar, apesar de ser muito controversa, ocorre nesta época, o que favorece a estimativa da idade das faixas etárias com implicações civis e criminais ${ }^{6,22}$.

Frente à imprecisão dos resultados iniciais na estimativa de indivíduos com mais de 15 anos, uma segunda avaliação foi feita analisando apenas segundos e terceiros molares, superiores e inferiores, objetivando a sensibilidade do teste para esta faixa etária. De fato, a nova metodologia trouxe uma frequência maior de acerto da idade real do indivíduo, só que às custas de um intervalo maior de estimativa, chegando a colocar a idade real do indivíduo num intervalo de 9 anos.

A análise da frequência de acerto de dentes isolados indicou que os terceiros molares apresentaram o maior índice de acerto quando comparado aos demais elementos dentários. Ennis \& Berry ${ }^{23}$, Ferreira ${ }^{24}$, Miles $^{25}$, Weise \& Bruntsch ${ }^{26}$ e Cornélio Neto ${ }^{27}$ desenvolveram estudos com os 3 os molares e concluíram que esse elemento é essencial e determinante para estimar a idade entre 14 e 22 anos. Porém, Samico ${ }^{28}$, em seu trabalho "O dente em sua evolução e a determinação da idade", chegou à conclusão de que não se deve ter confiança absoluta no 3ำ molar, pois este possui inúmeras variações evolutivas. Entretanto, devido ao maior percentual de acerto desse elemento, quando comparado aos demais, entre a idade estimada e a real, concluímos que os terceiros molares são os elementos mais indicados para se estimar a idade na faixa etária estudada, aliado a outros métodos de avaliação de idade.

Um método comumente utilizado para a estimativa da idade nos Institutos de Medicina Legal é o método visual. Este consiste na observação direta da presença ou ausência do terceiro molar na cavidade bucal, considerando o indivíduo maior de 18 anos com a presença desse elemento ou menor de 18 na ausência do mesmo. Porém este método é bastante falho, já que esse dente apresenta um padrão de erupção irregular e, muitas vezes nem tem espaço para irromper na cavidade oral, graças à evolução humana que veio acompanhada da diminuição do tamanho dos arcos dentários. O dente pode estar ausente também por ter sido extraído, por razões clínicas ou ortodônticas. Um método mais seguro seria o método radiográfico, pois com uma radiografia panorâmica é possível observar a mineralização de todos os dentes e através de um treinamento adequado e comparação com tabelas poderia se estimar com mais precisão a idade do indivíduo ${ }^{27,29}$.

Tendo em vista que, devido ao alto custo dos aparelhos de radiografias panorâmicas, provavelmente muitos dos Institutos Médico Legais do Brasil não possuem esse tipo de equipamento, as estimativas de idade ficam comprometidas. Uma alternativa viável seria a utilização de aparelhos de radiografias periapicais, tanto para exames em vivos como em mortos. Esse equipamento é vantajoso por ser de menor custo e possuir certa mobilidade, o que permitiria apenas um aparelho nas salas de necropsia. Além desses fatores, as tomadas radiográficas periapicais dos 3 os molares já seriam importantes para ajudar o Odontolegista a estimar a idade do indivíduo.

De maneira geral, a literatura demonstra que o amadurecimento esquelético ocorre mais cedo em mulheres do que homens ${ }^{21}$. Samico ${ }^{28}, \mathrm{Nolla}^{10}$, Arbenz $^{30}$, 
Nicodemo et al. ${ }^{16}$, Silva ${ }^{31}$, Kurita et al. ${ }^{19}$ e Kasper et al. ${ }^{32}$ concluíram que, sob o aspecto da cronologia da mineralização, os dentes dos indivíduos do sexo feminino apresentam precocidade, mais evidente na fase do término apical. Entretanto, Cornélio $\mathrm{Neto}^{27}$ trouxe o entendimento de que em homens, a mineralização dos seus $3^{\text {os }}$ molares, é mais precoce do que em mulheres, na faixa etária dos 104 aos 254 meses.

No presente estudo não foram encontradas diferenças significativas entre a cronologia de mineralização de ambos os sexos, estando de acordo com os resultados encontrados por Dantas ${ }^{33}$, Cruz et al. $^{34}$, Oliveira ${ }^{20}$, Lisbôa ${ }^{8}$. Os 3 os molares inferiores foram os únicos dentes com diferença significativa de mineralização quando comparados os grupos por gênero. Neste dente foi encontrada a maior diferença percentual de acertos, principalmente nas mulheres.

Com relação à utilização da tabela de Nicodemo, Moraes e Médici Filho ${ }^{16}$ em diferentes regiões brasileiras, um estudo realizado na cidade de Jequié$\mathrm{BA}^{34}$ demonstrou que o método utilizado para estimativa de idade forneceu valores compatíveis com a idade real dos indivíduos estudados. Em Belém- $\mathrm{PA}^{8}$ as médias de idade dos estágios iniciais de mineralização foram acima da média proposta na tabela, mas se igualaram a partir do estágio 7 de mineralização. Já Carneiro et al. ${ }^{35}$ ao estudar a população de Alagoas e Kurita et al. ${ }^{19}$ em Fortaleza, encontraram diferenças significativas entre as idades cronológicas e estimadas, sugerindo que o desenvolvimento de tabelas regionais de cronologia de mineralização dentária pode diminuir essa discrepância.

No Brasil, menores de 18 anos, após cometerem algum delito, não podem ter reclusões em sistemas carcerários comuns, pois são considerados imaturos segundo a teoria da formação mental incompleta. Atualmente, o tempo máximo de internação para adolescentes infratores em instituições correcionais é de $3 \operatorname{anos}^{36}$. Deste modo, em busca de sanções mais brandas, muitos indivíduos maiores de 18 anos alegam ser menores quando da responsabilização por conduta delituosa, o que aumenta a responsabilidade dos peritos ao estimarem a idade dessas pessoas. Neste contexto, esta pesquisa vem contribuir para que os odontolegistas tenham meios para realizar uma estimativa de idade mais fidedigna com a cronológica.

A determinação do estágio de mineralização dos dentes tem implicações importantes em estudos forenses relacionados à determinação de idade em pessoas vivas, principalmente no que tange a maioridade penal. No entanto, sua utilização metodológica como única técnica de estimativa de idade demonstra-se limitada, já que pode ter, como resultado, intervalos etários muito amplos. Todavia, quando associada a outros métodos como exame físico geral, raios-x do crânio, ossos longos e ombros, pode propiciar avaliação mais precisa da idade cronológica, precisão maior do que a oferecida por qualquer um dos métodos usados de forma isolada ${ }^{37}$.

\section{CONCLUSÕES}

Diante dos resultados obtidos e, seguindo o propósito do estudo, pode-se concluir que:

- Os terceiros molares apresentaram percentual maior de acerto na estimativa da idade quando comparados isoladamente com os demais dentes $(61,7 \%)$.

- No estudo não houve diferença significativa entre o período de mineralização dos elementos dentais quando comparados homens e mulheres. Quando da avaliação de 4 dentes de cada indivíduo ( $2^{\circ}$ e $3^{\circ}$ molares), a análise das radiografias das mulheres apresentou percentual mais elevado de acerto da idade cronológica do que a dos homens, sem contudo apresentar diferença estatisticamente significante. $\mathrm{Na}$ análise dos períodos etários, a faixa entre 10 e 15 anos apresentou $94,4 \%$ de acerto, todavia, a faixa acima de 15 anos exibiu valores estatisticamente diferentes da idade cronológica, muito embora este resultado deveu-se ao grande intervalo entre a idade máxima e mínima obtida pela amostra, sugerindo a fragilidade da utilização desta metodologia nesta população.

- A análise percentual da avaliação isolada dos $2^{\circ}$ e $3^{\circ}$ molares por sexo demonstrou percentuais discretamente maiores para mulheres, no entanto, apresentou diferença estatística apenas na avaliação dos 3 os molares inferiores.

- Os métodos de estimativa da idade utilizando-se a tabela de Nicodemo, Moraes e Médici Filho, propostos por este estudo, demonstraram percentuais baixos de acerto com relação à idade cronológica dos indivíduos, quando a avaliação foi realizada com 16 dentes. Já avaliação de 4 dentes apresentou resultados que demonstraram ser a metodologia mais satisfatória, principalmente para análise da faixa entre 10 e 15 anos. - Ao se buscar a estimativa de idade como técnica para responder à solicitação da Justiça, os Odontolegistas devem associar seus resultados ao de outras técnicas para o mesmo fim, ampliando, assim, sua acuidade. 
Moreno MBP et al. Utilização da Tabela de cronologia de mineralização dental de Nicodemo, Moraes e Médici Filho.

Moreno MBP; Pontes TJP; Rabello PM. The use of the Nicodemo, Moraes e Médici Filho Table of dental mineralization chronology to estimate the Paraibanos' age. Saúde, Ética \& Justiça. 2014;19(1);35-44.

ABSTRACT: Objective: To verify the chronological age hit rate of young people using the Nicodemo, Moraes and Medici Filho table. Methods: The sample consisted of 94 panoramic radiographs images of individuals aged 10 to 25 years. The mineralization table described in NOLLA's study was used according to the chronological table of permanent teeth mineralization in Brazil which was created by Nicodemo, Moraes and Medici Filho. The research method was transversal and blind. For the statistical analysis absolute and percentage distributions (Descriptive statistical techniques) were obtained and the chi-square test (inferential statistical techniques) was used. The significance level used in the statistical tests was 5\%. Results: The hit percentage in the age group described was higher when only four teeth were analyzed by panoramic radiography $(81.9 \%)$ compared to the method of Nolla $(4.3 \%)$. The third molars had the highest percentage of correct answers $(66 \%)$ and the hit percentage was higher among individuals aged $10-15$ years $(94.4 \%)$ than those who were older than 15 years (65\%). Conclusion: The Nolla's method modified by Nicodemo, Moraes and Medici Filho was not effective for the age group studied, and in these cases the observation of the 3rd molar mineralization, in isolation, would be of great value and could help the forensic dentist in the estimated age of the individual.

KEYWORDS: Forensic Dentistry; Age Determination by Teeth; Radiology.

\section{REFERÊNCIAS}

1. Vanrell JP. Odontologia Legal e Antropologia Forense.

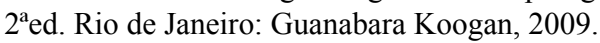

2. Gonçalves ACS, Antunes JLF. Estimativa da idade em crianças baseada nos estágios de mineralização dos dentes permanentes com finalidade odontolegal. Odontologia e Sociedade. 1999; 1(1/2):55-62. Disponível em: http://143.107.240.24/departamentos/social/legal/arquivos/ estimativa_da_idade.pdf

3. Schmidt CM. Estimativa da idade e sua importância forense [dissertação]. São Paulo: UNICAMP, Faculdade de Odontologia de Piracicaba; 2004. Disponível em: $\quad$ http://www.bibliotecadigital.unicamp.br/ document/?code $=$ vtls000322790

4. Schmeling A, Geserick G, Reisinger W, Olze A. Age estimation. Forensic Science International. 2007; 165(2/3):178-81. DOI: http://dx.doi.org/10.1016/j. forsciint.2006.05.016

5. Costa FE. Estimativa de idade em radiografias panorâmicas através dos estágios de calcificação de Nolla [dissertação]. São Paulo: UNICAMP, Faculdade de Odontologia de Piracicaba; 2001. Disponível em: http://www. bibliotecadigital.unicamp.br/document/?code $=$ vtls 000226 $235 \& \mathrm{fd}=\mathrm{y}$

6. Cordeiro RCL, Pinto, LAMS, Gonçalves MA, Mendes AJD. Etapas da formação e mineralização do terceiro molar em crianças: estudo radiográfico. Rev Odontol. UNESP, São Paulo. 1999; 28(2):401-14. Disponível em: http:// www.revodontolunesp.com.br/files/v28n2/v28n2a13.pdf

7. Carvalho TAA. Mineralização dentária na estimativa da idade do indivíduo [monografia]. João Pessoa: Universidade Federal da Paraíba; 2009. Disponível em: http://www.ccs. ufpb.br/dor/templates/joomla-vortex/TCC/09.2/21.pdf

8. Lisbôa MBM. Radiografia panorâmica no contexto da segurança pública: estimativa de idade no caso de Belém/ PA [dissertação]. Belém: Universidade Federal do Pará; 2013. Disponível em: http://www.ppgdsmc.ufpa.br/docs/ dissertacoes/Disserta-Maria-Betania-Lisboa.pdf
9. Saliba JHM. Estudo radiográfico comparativo entre a cronologia de mineralização dentária e a maturação óssea das vértebras cervicais [dissertação]. Três corações: Universidade Vale Do Rio Verde; 2005. Disponível em: http://www.dominiopublico.gov.br/pesquisa/ DetalheObraForm.do?select_action $=\&$ co_obra $=41746$

10. Nolla CM. The development of the permanent teeth. J Dent Child. Fulton, 1960; 27:254-66.

11. Demirjian A, Goldstein H, Tanner,JM. A new system of dental age assessment, Human Biology. 1973; 45(2):21127. Disponível em: http://www.bristol.ac.uk/cmm/team/hg/ full-publications/1973/dental-age-assessment.pdf

12. Koshy S, Tandon, S. Dental age assessment: the applicability of Demirjian's method in south Indian children. Forensic Sci Int. 1998; 94(1/2):73-85. DOI: http://dx.doi.org/10.1016/ S0379-0738(98)00034-6

13. Liversidge HM, Herdeg B, Rösing FW. Dental age estimation of non-adults. A review of methods and principles. In: Alt WK, Rösing FW, Teschler-Nicola M (eds). Dental anthropology. Fundamentals, limits, and prospects. Springer: Wien New York; 1998. p 419-44.

14. Liversidge,HM. Accuracy of age estimation from developing teeth of a population of known age (0-5.4 years). Int $\mathrm{J}$ Osteoarchaeol. 1994; 4(1):37-45. Disponível em: http:// onlinelibrary.wiley.com/doi/10.1002/oa.1390040107/pdf

15. Gustafson G, Koch G. Age estimation up to 16 years of age on dental development. Odontol Revy. 1974; 25(3):297306.

16. Nicodemo RA, Moraes LC, Médice Filho E. Tabela cronológica da mineralização dos dentes permanentes, entre brasileiros. Rev Fac Odontol. 1974; 3(1):55-6.

17. IBGE. Síntese de indicadores sociais. Uma análise das condições de vida da população brasileira. Rio de Janeiro; 2010. Disponível em: http://www.ibge.gov.br/home/ estatistica/populacao/condicaodevida/indicadoresminimos/ sinteseindicsociais2010/SIS_2010.pdf

18. Meinl A, Tangl S, Huber C, Maurer B, Watzek G. The 
chronology of third molar mineralization in the Austrian population - a contribution to forensic age estimation. Forensic Science International. 2007; 169(2/3):161-7. DOI: http://dx.doi.org/10.1016/j.forsciint.2006.08.014

19. Kurita LM, Menezes AV, Casanova MS, Haiter-Neto F. Dental maturity as an indicator of chronological age: radiographic assessment dental age in a brazilian population. J Appl Oral Sci. 2007; 15(2):99-104. DOI: http://dx.doi.org/10.1590/S1678-77572007000200005

20. Oliveira FT. Estimativa da idade cronológica por meio de avaliação radiográfica da mineralização de terceiros molares e altura do ramo da mandíbula [tese]. São Paulo: USP, Faculdade de Odontologia de Bauru; 2010. Disponível em: http://www.teses.usp.br/teses/disponiveis/25/25132/ tde-02062011-095629/pt-br.php

21. Mendes YBE, Bergmann JR, Pellissari MF, Hilgenberg SP, Coelho U. Analysis of skeletal maturation in patients aged 13 to 20 years by means of hand wrist radiographs. Dental Press J Orthod. 2010; 15(1):74-9. Disponível em: http:// www.scielo.br/pdf/dpjo/v15n1/en 09.pdf

22. Silva RF, Daruge Júnior E, Pereira SDR, Almeida SM, Oliveira RN. Identificação de cadáver carbonizado utilizando documentação odontológica. Rev odonto ciênc. 2008; 23(1):90-3. Disponível em: http://revistaseletronicas. pucrs.br/ojs/index.php/fo/article/viewFile/1245/2881.

23. Ennis LM, Berry HM. Dental roentgenology. $3^{\mathrm{a}} \mathrm{ed}$. Philadelphia: Lea \& Febiger; 1939.

24. Ferreira AA. Da técnica médico-legal na investigação forense. $2^{\mathrm{a}}$ ed. São Paulo: Revista dos Tribunais; 1962.

25. Miles AEW. Dentition in the estimation of age. J Dent Res. Washington, 1963; 42: 255-63. DOI: http://dx.doi.org/10.1 177/00220345630420012701

26. Weise W, Bruntsch E. Roentgenographic studies regarding the development and presence of the third molar. Azhnarztl Radsch. Berlin. 1965; 74(6):206-16.

27. Cornélio Neto WL. Estimativa da Idade pela mineralização dentária dos $3^{\circ}$ molares através de radiografias panorâmicas [dissertação]. São Paulo: UNICAMP, Faculdade de Odontologia de Piracicaba; 2000. Disponível em: http://www.bibliotecadigital.unicamp.br/ document $/$ ?code $=$ vtls000205991

28. Samico A. O dente em sua evolução e a determinação da idade [tese]. Recife: Faculdade de Direito do Recife, 1943.

29. Ferreira FV, Diagnóstico em Ortodontia. São Paulo: Artes Médicas; 2002.

30. Arbenz GO. Compêndio de medicina legal, São Paulo: Atheneu;1983.

31. Silva M. Compêndio de odontologia legal. São Paulo: Medsi; 1997.

32. Kasper KA, Austin D, Kvanli AH, Rios TR, Senn DR. Reliability of third molar development for age estimation in a Texas Hispanic population: a comparison study. J Forensic. 2009; 54(3): 651-7. DOI: http://dx.doi.org/10.1111/j.15564029.2009.01031.x

33. Dantas RMX. Correlação entre idade cronológica e mineralização dos terceiros molares em uma amostra populacional do nordeste brasileiro [monografia]. João Pessoa: Universidade Federal da Paraíba; 2010. Disponível em: http://www.ccs.ufpb.br/dor/templates/joomla-vortex/ TCC/09.2/12.pdf

34. Cruz JPP, Duque Neta DG, Lantyer JS, Da Silva ACC, Barros GB. Estimativa da idade através dos estágios de mineralização dentária em crianças e adolescentes de Jequié-BA. Anais do I Encontro de Odontologia Legal de Ribeirão Preto. Ribeirão Preto, SP; 2010.

35. Carneiro APC, Guimarães JATL, Silva RMI, Santiago, APCS, Laureano Filho JR. Chronological table of third molar mineralization in a survey in the state of Alagoas, Brazil. Braz J Oral Sci. 2010; 9(4):488-92. Disponível em: http://revodonto.bvsalud.org/scielo.php?script=sci arttext\&pid=S1677-32252010000400014\&lng=pt\&nrm $=$ is so

36. Brasil. Lei n. 8.069, de 13 de julho de 1990. Dispõe sobre o Estatuto da Criança e do Adolescente e dá outras providências. Disponível em: http://www.planalto.gov.br/ ccivil_03/leis/18069.htm

37. Braude SC, Henning LM, Lambert MI. Accuracy of bone assessments for verifying age in adolescents - application in sport. SA Journal of Radiology. 2007; 11(2):4-7. Disponível em: http://www.ajol.info/index.php/sajr/article/view/34492 